\title{
SÍNDROME DE TAKOTSUBO EN EL INSTITUTO SALVADOREÑO DEL SEGURO SOCIAL
}

\author{
Griceyda B. Medrano \\ Wendy J. Sandoval ${ }^{2}$ \\ Recibido 09/10/19 \\ Aceptado 20/04/20
}

\section{RESUMEN}

El síndrome de Tako-Tsubo fue descrito por primera vez en 1990 por Sato et al. Se caracteriza por la hipocinesia o acinesia apical transitoria del ventrículo izquierdo y coronarias normales por cateterismo. Se presenta un caso clínico de una paciente femenina ingresada con cuadro sospechoso de síndrome coronario posterior a desencadenante emocional, que cumple criterios propuestos por la clínica Mayo 2008 para el diagnóstico de Síndrome Tako-Tsubo.La importancia de identificar este síndrome radica en que su presentación clínica simula un infarto agudo del miocardio; sin embargo, su pronóstico y morbimortalidad son diferentes.

Palabras clave: Síndrome de Takotsubo, miocardiopatia por estrés. 


\section{INTRODUCCIÓN}

Se trata de paciente femenina de 71 años de edad quien ingreso al Hospital General del Instituto Salvadoreño del Seguro Social (ISSS) con sospecha de infarto agudo del miocardio sin elevación del ST, concluyéndose que se trataba de un Síndrome de Takotsubo.

Síndrome de Takotsubo llamado también síndrome de discinecia apical transitoria fue descrito por primera vez en Japón en los años 90. Entidad poco frecuente. Se caracteriza por dolor precordial anginoso, cambios electrocardiográficos, elevación de enzimas de daño miocárdico, ausencia de obstrucción coronaria en la angiografía y una característica discinecia anteroapical del ventrículo izquierdo que se normaliza en unos pocos días. El estrés emocional severo es el desencadenante más común. La etiopatogenia de este síndrome está aún por definir. Establecido por los siguientes criterios diagnósticos (1). (Tabla 1)

\section{Criterios diagnósticos de la Clínica Mayo (2008) para síndrome de Tako-Tsubo}

1. Alteraciones transitorias en la contractilidad ventricular izquierda (discinesia, acinesia o hipocinesia), con afectación apical o sin ella; extendiéndose más allá del territorio de una arteria coronaria determinada; ocasionalmente hay una situación estresante desencadenante, aunque no siempre

2. Ausencia de enfermedad coronaria obstructiva o evidencia angiográfica de rotula de placa aguda

3. Nuevas alteraciones electrocardiográficas (elevación de ST y/o inversión de la onda T) o elevación modesta de troponina

\section{Ausencia de feocromocitoma o miocarditis}

Tabla 1. Criterios diagnósticos de la clínica Mayo.

Epidemiologia: varios estudios estadounidenses han estimado que representan el 1 al $2 \%$ de los síndromes coronarios agudos con onda $\mathrm{Q}$ sin elevación del ST respectivamente. La edad pico de los pacientes es 69 años; la edad media varía entre los
58 y los 75 años (2). Un 82-100 \% de los casos se da en mujeres postmenopáusicas con pocos factores de riesgo cardiovascular.

Se desconoce la razón del predominio femenino, atribuyéndose a la deprivación de estrógenos, que puede influir en el sistema nervioso simpático y afectar a la reactividad vascular coronaria o tendencia al espasmo.

Es posible que la disfunción endotelial, que empeora tras la menopausia, incremente la vulnerabilidad al aturdimiento miocárdico mediado simpáticamente.

En un $50 \%$ de casos se ha identificado la hipertensión arterial como factor de riesgo asociado. Hasta en el $75 \%$ de los casos es precedido de un factor estresante emocional o físico (3). (Tabla

\begin{tabular}{|l|l|}
\hline $\begin{array}{l}\text { Estresantes } \\
\text { emocionales }\end{array}$ & Estresantes Físicos \\
\hline $\begin{array}{l}\text { Muerte inesperada de } \\
\text { pariente o amigo }\end{array}$ & $\begin{array}{l}\text { Exacerbación de } \\
\text { trastornos sistémicos }\end{array}$ \\
\hline Abuso doméstico & $\begin{array}{l}\text { Procedimiento } \\
\text { invasivos no cardíacos }\end{array}$ \\
\hline Confrontación & $\begin{array}{l}\text { Esfuerzos físicos } \\
\text { extenuantes }\end{array}$ \\
\hline $\begin{array}{l}\text { Diagnóstico médico } \\
\text { catastrófico }\end{array}$ & Ataque de asma \\
\hline Negocio devastador & Neumotórax \\
\hline Fiesta sorpresa & Fibrilación ventricular \\
\hline Reunión sorpresa & Exposición al frío \\
\hline Accidente de coche & \\
\hline Miedo al procedimiento & \\
\hline Robo a mano armada & $\begin{array}{l}\text { Comparecencia en } \\
\text { tribunal }\end{array}$ \\
\hline Actuación en público & \\
\hline
\end{tabular}

Tabla 2. Factores descencadenantes.

Fuentes. Tsuchihashi et al. ${ }^{4}$ y Bybee et al . ${ }^{7}$ 
Etiopatogenia: se cree que altos niveles de epinefrina circulante es la señal disparadora intracelular en el cardiomiocito ventricular por acción de los receptores B-adrenérgicos.

La explicación fisiopatología se concentra en un efecto directo de altos niveles de epinefrina en el miocardio ventricular, tiene predilección por el miocardio apical (4).

Cuadro clínico: Síndrome coronario agudo. El electrocardiograma inicial puede ser similar al del síndrome coronario agudo, con elevación del segmento ST en precordiales, ondas T negativas en precordiales y onda $\mathrm{Q}$, siendo rara la imagen en cara inferior. (Tabla 3)

1. Inicio súbito de dolor torácico o alteración de la respiración

2. Estresante físico o emocional actuando como factor precipitante

3. Predominante en mujeres postmenopáusicas

4. Cambios electrocardiográficos indicativos de infarto agudo de miocardio

5. De mínima a moderada elevación de biomarcadores cardíacos

6. Acinesia o hipocinesia apical y medioventricular transitoria del ventrículo izquierdo, con hipercinesia basal

7. Típica forma de recipiente para la recogida de pulpos en la ecocardiografía o ventriculografía

8. Ausencia de lesiones coronarias en la coronariografía

9. Ausencia de otros estados catecolaminérgicos como feocromocitoma, traumatismo craneoencefálico con sangrado intracraneal agudo, etc.

10. Completa resolución de la disfunción ventricular izquierda a las pocas semanas del inicio del cuadro

Tabla 3. Características clínica.
Ecocardiograma: Inicialmente muestra una discinecia o acinesia del ápex del ventrículo izquierdo, con normo o hipotcinesia basal. La repetición tras la fase aguda confirmará que las anomalías han revertido (3).

Coronariografía: no muestra lesiones coronarias. En la ventriculografía se observa una disfunción del ventrículo izquierdo con hipocinesia, acinesia o discinesia de los segmentos apicales con hipercontractilidad de las bases, dando como resultado la balonización durante la sístole (3). (Figura 6)
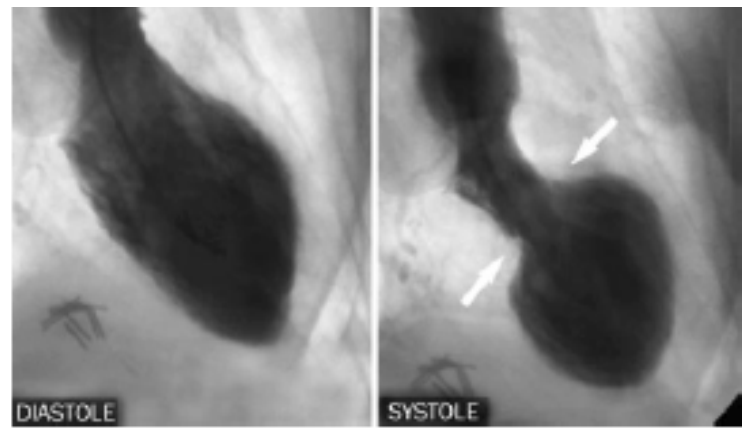

Figura 6. Hipercontractilidad basal, con acinesia apical y abalonamiento.

La forma distintiva incluye una porción superior o cuello, en contraste con una porción elongada baja, que recuerda a la cerámica Japonesa "takotsubo" empleada para capturar pulpos (5). (Figura 7)

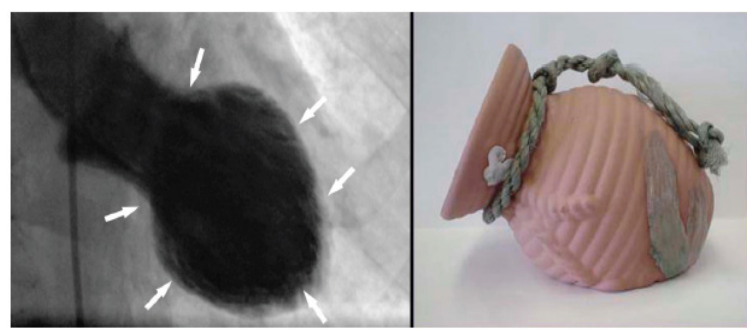

Figura 7. Cerámica utilizada para atrapar pulpos.

Evolución: La patología es reversible y la recuperación usualmente es rápida y completa. La función cardiaca contráctil mejora gradualmente en días, siendo normal en 7 días promedio; sin embargo, puede llevar varias semanas (3). 
Complicaciones: Generalmente ocurren durante la fase aguda, siendo raras en fase tardía porque es reversible. La tasa de complicaciones es del $19 \%$. El fallo cardíaco y el edema pulmonar van del 3 al $46 \%$ de los pacientes y la tasa de mortalidad es del 17 al $3 \%$.

Otras: obstrucción dinámica del VI secundaria a un gradiente de presión, regurgitación mitral, arritmias ventriculares, bloqueo auriculoventricular agudo transitorio, formación de trombos intramurales en el ventrículo izquierdo, accidente cerebrovascular de origen embólico, rotura de la pared libre del VI y muerte (3).

Tratamiento: Inicialmente es indistinguible del SCA, el tratamiento inicial será de la isquemia coronaria con monitorización, antiagregación y/o anticoagulación, oxigenoterapia, inhibidoresde la glucoproteína iib/iiia, y/o anticoagulantes, nitratos, betabloqueantes, Inhibidores de la enzima convertidora de angiotensina y diuréticos.

En vista que es una entidad clínica poco frecuente y muchas veces subdiagnosticada. Es importante su estudio y publicación como parte de diagnóstico diferencial del síndrome coronario agudo, particularmente en nuestro medio donde no hay una descripción de esta enfermedad, ni estudio de caso.

\section{Caso clínico}

Paciente femenina de 71 años, diabética e hipertensa de 15 años de evolución tratada con enalapril 20 mg vía oral cada día más metformina 850 mg media tableta vía oral cada día.

Consulto el 27 mayo 2014 con historia de 2 días de evolución de presentar dolor centro torácico opresivo irradiado a cuello y mandíbula, acompañado de cotejo vagal. Por lo que evaluaron en unidad de emergencia encontrándose: FC: 90 lpm, FR: $16 \mathrm{rpm}$, TA: $110 / 84 \mathrm{mmHg}, \mathrm{T}^{\circ} 37^{\circ} \mathrm{C}$, Sat $\mathrm{O} 2$ 99 \%, HGT: 116 mg/dl.No hallazgos al examen físico por sistemas. Se tomó EKG inicial mostrando inversión de onda T en derivaciones V3 - V6 mas por lo que ingresan con sospecha de Síndrome Coronario Agudo sin elevación del ST (Figura 1). Se realizan los siguientes exámenes. (Tabla 4)

\begin{tabular}{|l|l|l|}
\hline Química & $27 / 06 / 14$ & $28 / 06 / 14$ \\
\hline CPK T & 226 & 184 \\
\hline CK MB & 16 & 20 \\
\hline LDH & 163 & 158 \\
\hline GLUCOSA & 121 & \\
\hline CR & 0.6 & \\
\hline UN & 7.1 & \\
\hline UREA & 14 & \\
\hline NA & 132 & \\
\hline K & 4 & \\
\hline & Hemograma \\
\hline GB & 6390 \\
\hline N & $74 \%$ & \\
\hline L & $11.5 \%$ \\
\hline HB & 10.9 \\
\hline HTO & 31.7 \\
\hline PLAQUETAS & 354000 \\
\hline
\end{tabular}

Tabla 4. Exámenes De Laboratorio.

Evaluada por cardiólogo quien indica ECO 2 y Coronariografía.

Se realiza primer ECO 2-D el día 27/05/14 (Figura 2) que reporta aquinesia septal segmento medio y apical contractilidad conservada en el resto de segmentos, función sistólica disminuida FE 45\% insuficiencia mitral y tricuspidea moderada PSAP: $41 \mathrm{mmHg}$, no trombos.

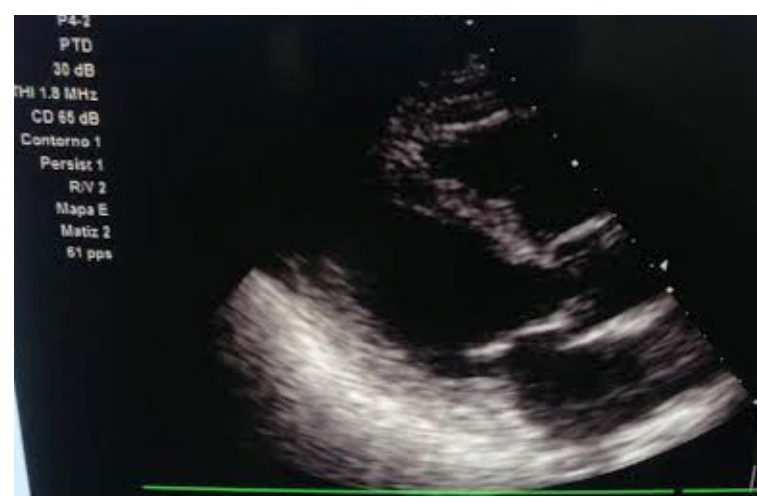

Figura 2. ECO 2 D 27/05/14 

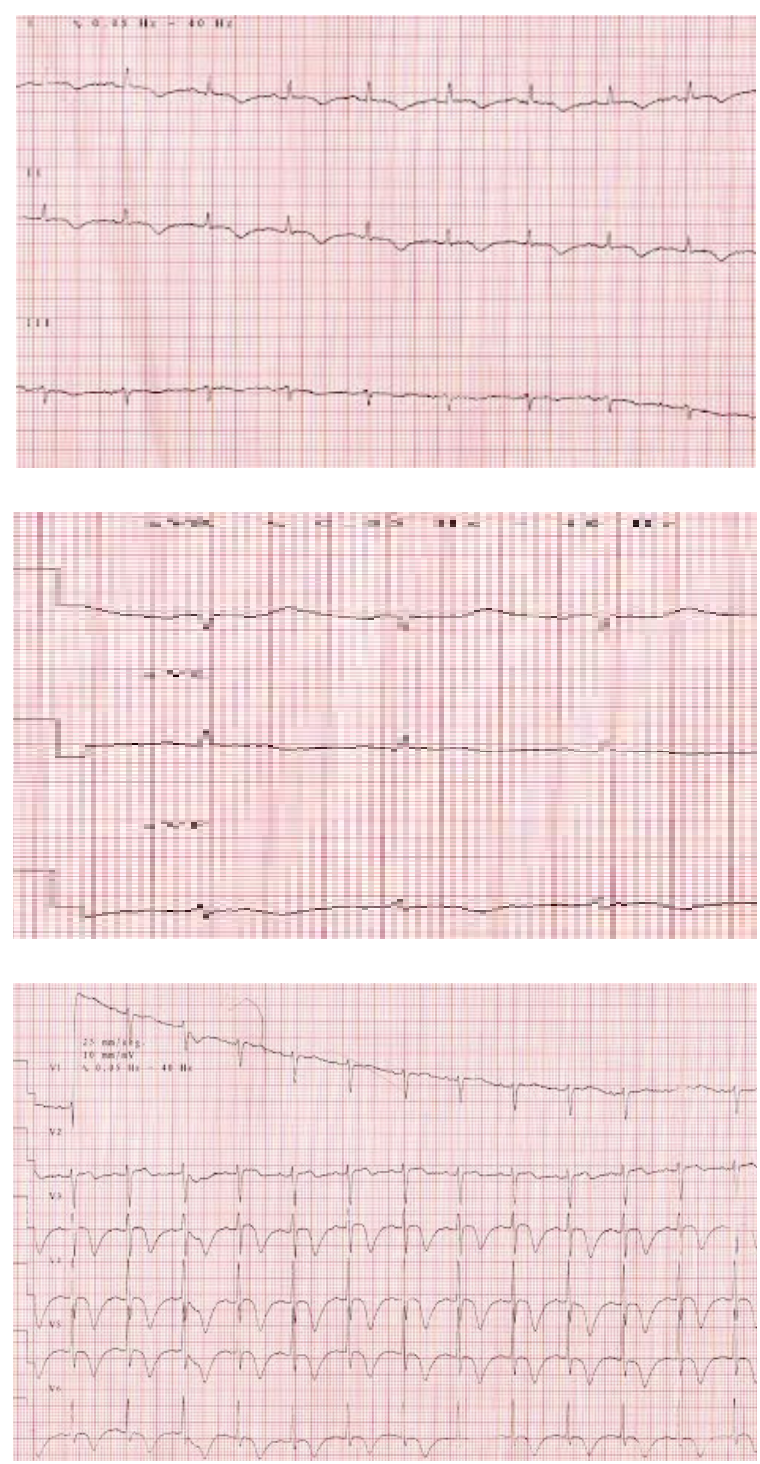

EKG \#1 INGRESO 27/05/14

El día 30/05/14 se realiza coronariografia diagnostica (figura 3) reportando: no lesiones angiográficas, insuficiencia mitral ligera a moderada. $1 / 4$ de hipocinesia apical con hiperquinesia apical.
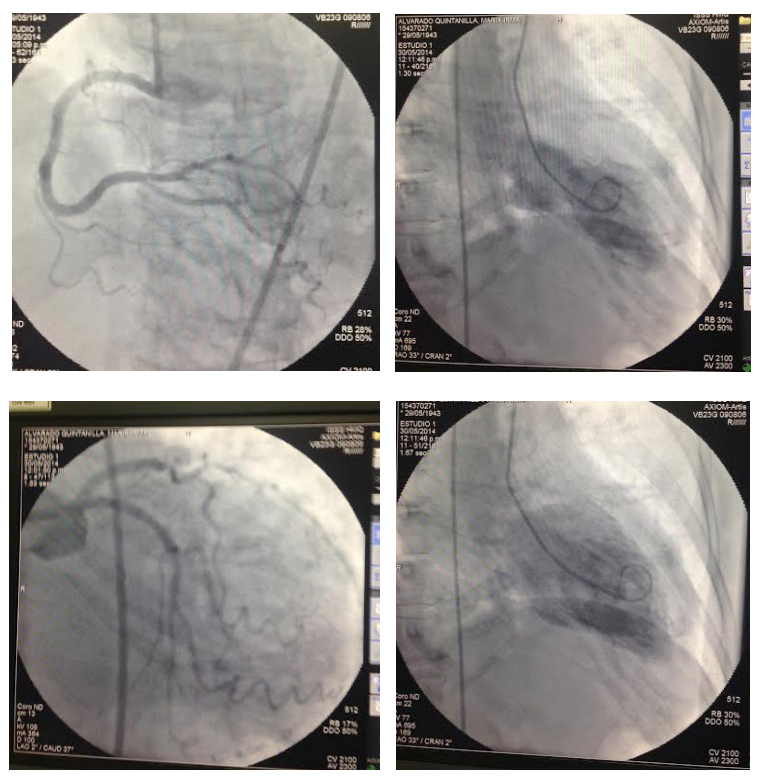

Figura 3. Coronariografia con ventriculografia.

Cumplió tratamiento de Síndrome Coronario Agudo. Es reevaluada por cardiología nuevamente 04/06/14 quien al correlacionar datos obtenidos y cuadro clínico se orienta patología por miocardiopatia por estress, por lo que ante la sospecha se toma nuevo ECO 2D de control (Figura 4) encontrando los siguientes hallazgos: hipertrofia concéntrica del ventrículo izquierdo, contractilidad y función sistólica normal, FE 69\%, aortoesclerosis e insuficiencia aortica leve. Insuficiencia mitral y tricuspidea, ambas leves. Pericardio normal.

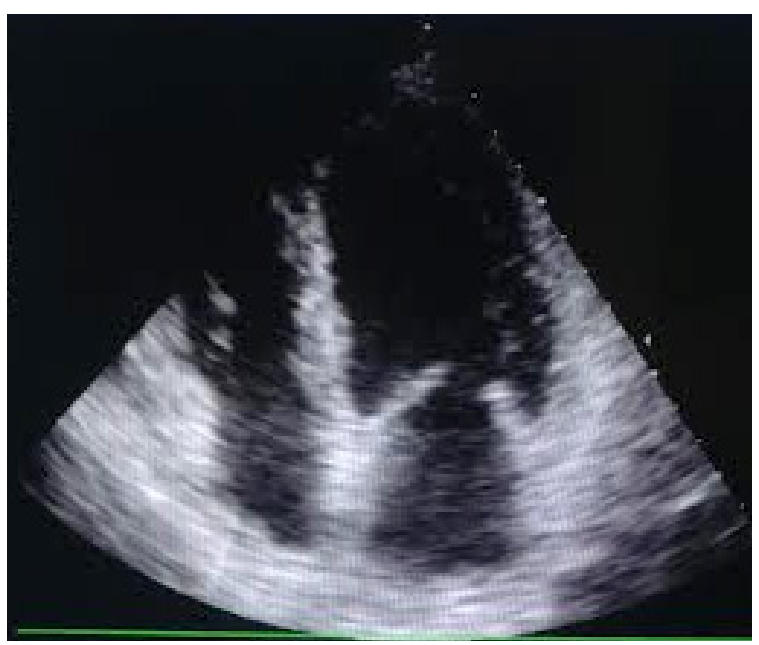

Figura 4. ECO 2D \#2. 04/06/2014 
EVOLUCIÓN: Paciente recuperó buena parte de FE en ECO 2D control, cumplió tratamiento de Síndrome Coronario Agudo, anticoagulación con Enoxaparina a $1 \mathrm{mg} / \mathrm{kg}$ cada 12 horas, se instauró tratamiento con atorvastatina $80 \mathrm{mg}$ vo cada día, Ácido Acetil Salicilico $100 \mathrm{mg}$ vo cada día, clopidogrel $75 \mathrm{mg}$ vo cada dia, carvedilol $6.25 \mathrm{mg}$ vo cada día y enalapril $20 \mathrm{mg}$ vo cada día.

\section{EVOLUCIÓN ELECTROCARDIOGRÁFICA}
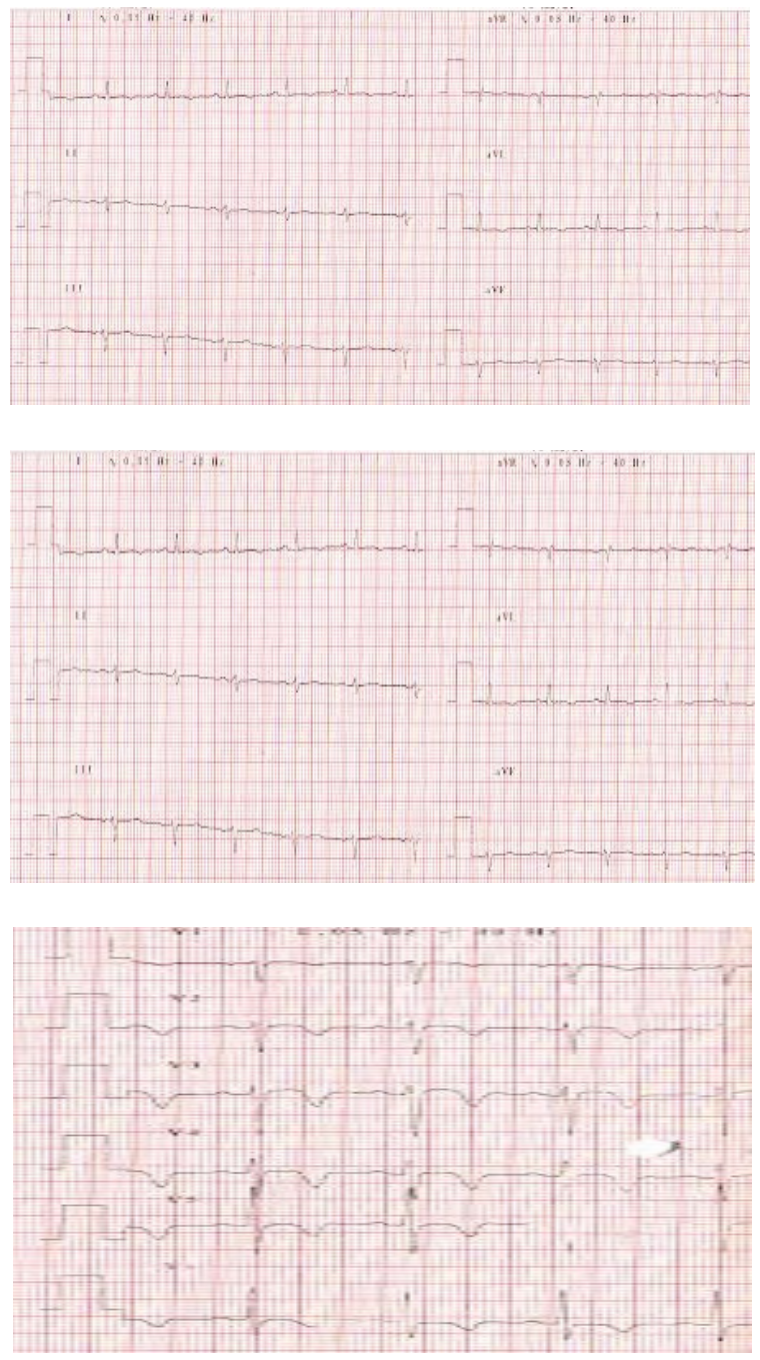

EKG \#2 05/06/2014
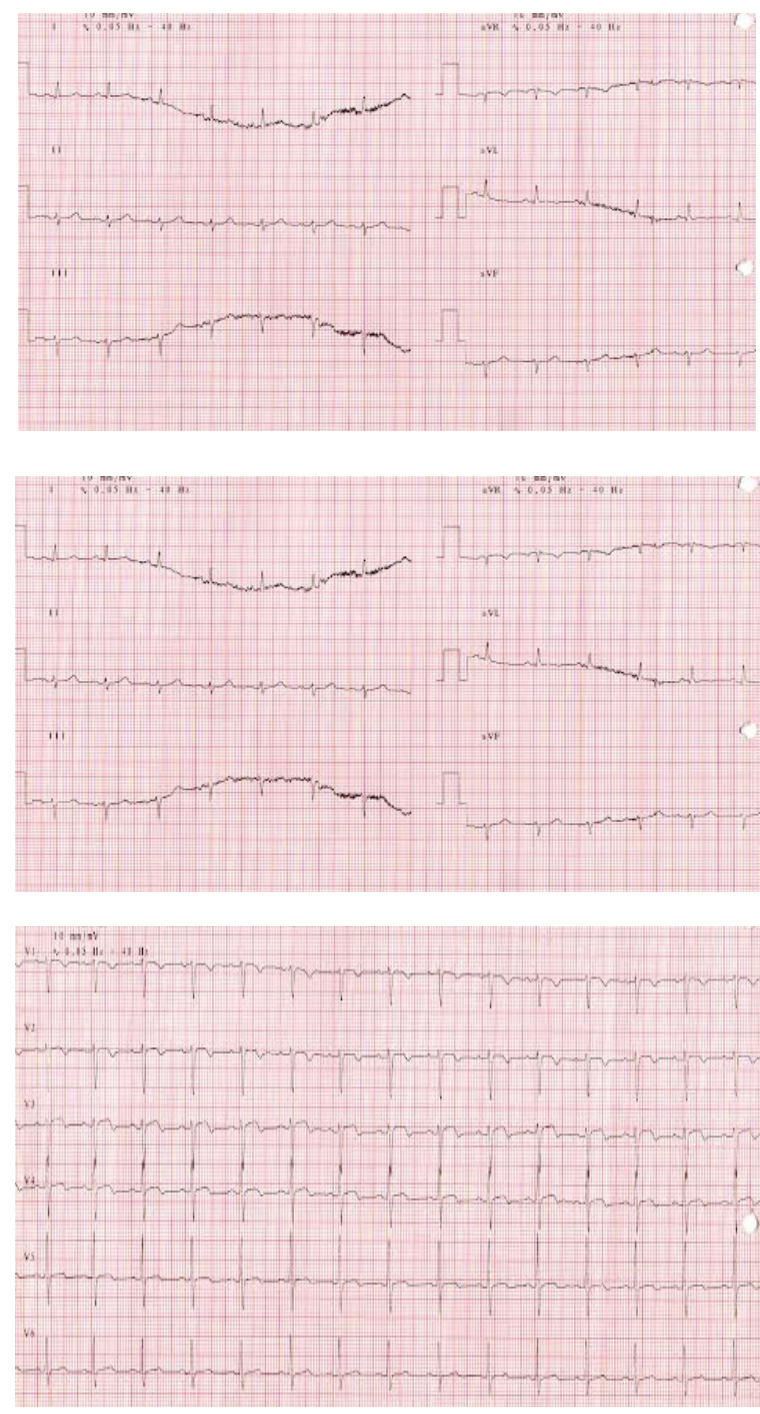

EKG \#3 Tomado el 08/06/2014

\section{DISCUSIÓN}

Esta enfermedad descrita pero con pocos casos publicados tiene trascendencia relevante como parte de diagnóstico diferencial del síndrome coronario agudo.

Se presenta caso clínico documentado en el Hospital General del Instituto Salvadoreño del Seguro Social (ISSS). 
Mujer en la octava década de la vida con cuadro de dolor torácico típico, quien ingresa con cuadro clínico y electrocardiográfico compatible con síndrome coronario, marcadores enzimáticos negativos (sin disponibilidad de troponina al ingreso ) en ECO $2 \mathrm{D}$ acinesia septal segmento medio y apical y $\mathrm{FE}$ $45 \%$, realizando coronariogradia sin lesiones angiográficas, apoyando la sospecha de un síndrome de Tako-Tsubo, motivo por el cual se dio seguimiento a este caso, con ECO 2 D control en el cual se demostró recuperación de la FEVI 69 \% con contractibilidad y movilidad normal. Evidenciándose en secuencia electrocardiografica cabios en la onda $\mathrm{T}$ en derivaciones precordiales cara antero-lateral.
Paciente completo tratamiento en Unidad de cuidados intensivos con buena evolución clínica.

El síndrome de Tako-Tsubo es una entidad clínica que sorprende por su presentación muy similar al síndrome coronario agudo; sin embargo, con mejor evolución y pronóstico.

La importancia de dar a conocer casos poco usuales en nuestra práctica diaria hace pensar que quizá el padecimiento es más frecuente de lo esperado en nuestro medio y que se encuentra subdiagnosticado o confundiéndose con el síndrome coronario agudo, lo que sugiere poner más énfasis en la realización de cateterismo cardiaco con ventriculografía para el diagnóstico deferencial. 


\section{Fuentes de información consultadas}

1. Prasad A. Apical ballooning syndrome: an important differential diagnosis of acute myocardial infarction. Circulation 2007;115:e56-9.

2. Cardiopatía de estrés o síndrome de TakoTsubo: conceptos actuales Iván J. Núñez Gil 1, María Luaces Méndez 2, Juan C. García-Rubira 1 REV ARGENT CARDIOL 2009;77:218-223.

3. Síndrome de tako-tsubo. Discinesia transitoria del ventrículoizquierdoF.M. Pérez Péreza,*y J. Sánchez Saladoba Servicio de Emergencias, Empresa Pública de Emergencias Sanitarias de Andalucía, Servicio Provincial de Cádiz, Cádiz, Espa nabServicio de Enfermería, Empresa Pública de Emergencias Sanitarias de Andalucía, Servicio Provincial de Cádiz, Cádiz, España. 31 de enero de 2013
4. Stress (Takotsubo) cardiomyopathy - a novel pathophysiological hypothesis to explain catecolamine - induced actue myocardical stunning Alexander R Lyon*, Paul SC Rees, Sanjay Prasad, Philip A Poole-Wilson and Sian E Harding, january 2008 vol 5 no 1.

5. Takotsubo (stress) Cardiomyopathy. Scott W. Sharkey, John R. Lesser and Barry J. Marron. Circulation. May 6, 2014

6. Takotsubo Cardyomiopathy, or Broken Heart Syndrome. Case serie, Volume 34, Number 1, 2007. Salim S. Virani, MD. A. Nasser Khan, MD. Cesar E. Mendoza, MD. Alexandre C. Ferreira, MD. Eduardo de Marchena, MD

7. Actue y Reversible Cardiomyopathy Provoked by Stress in Woman From the united States. Scott W. Sharkey, Andrew G. Zenovick, John R. Lesser and Barry J. Marron. Circulation. May 14,2014 